\title{
Superconductivity in the Penson-Kolb Model on a Triangular Lattice
}

\author{
A. Ptok And M. Mierzejewski \\ Institute of Physics, University of Silesia \\ Uniwersytecka 4, 40-007 Katowice, Poland
}

\begin{abstract}
We investigate properties of the two-dimensional Penson-Kolb model with repulsive pair hopping interaction. In the case of a bipartite square lattice this interaction may lead to the $\eta$-type pairing, when the phase of superconducting order parameter changes from one lattice site to the neighboring one. We show that this interaction may be responsible for the onset of superconductivity also for a triangular lattice. We discuss the spatial dependence of the superconducting order parameter and demonstrate that the total momentum of the paired electrons is determined by the lattice geometry.
\end{abstract}

PACS numbers: 74.20.-z, 74.20.Rp

\section{Introduction}

The Penson-Kolb model [1] can be derived from a general microscopic tight-binding Hamiltonian. In such approach, the Coulomb repulsion leads to the repulsive pair hopping interaction $J$. However, the pair hopping integral may also be considered as the effective model parameter that takes on both positive and negative values $[2-4]$. In the case of a square lattice the superconducting correlations occur independently of the sign of $J$. Superconductivity that occurs for repulsive pair hopping interaction is usually referred to as $\eta$-type pairing. In this phase the total momentum of the paired electrons is $(\pi, \pi)$. As a result, the original translational invariance is broken, and the superconducting order parameter alters from one site to the neighboring one. Although, the $\eta$-pairing is robust against the diamagnetic pair-breaking [5], the flux quantization and the Meissner effect appear in this state [6].

Here, we demonstrate that the repulsive pair hopping interaction may lead to a stable superconducting phase also on a non-bipartite triangular lattice. 


\section{Model and details of calculations}

We investigate the two-dimensional Penson-Kolb model

$$
H=-t \sum_{\langle i, j\rangle, \sigma} c_{i \sigma}^{\dagger} c_{j \sigma}+J \sum_{\langle i, j\rangle} c_{i \uparrow}^{\dagger} c_{i \downarrow}^{\dagger} c_{j \downarrow} c_{j \uparrow}-\mu \sum_{i, \sigma} c_{i \sigma}^{\dagger} c_{i \sigma},
$$

where $c_{i \sigma}^{\dagger}$ creates an electron with spin $\sigma$ on site $i$ and $\mu$ is chemical potential. We assume the repulsive pair hopping interaction $J>0$.

We apply the mean-field approximation and introduce $\Delta_{i}=\left\langle c_{i \downarrow} c_{i \uparrow}\right\rangle$. As the superconductivity induced by positive $J$ may break the original translational invariance, $\Delta_{i}$ should be considered as a site dependent quantity. In the case of $\eta$-pairing on square lattice one gets

$$
\Delta_{i}=\Delta_{0} \exp \left(\mathrm{i} \boldsymbol{Q} \cdot \boldsymbol{R}_{i}\right)
$$

with $\boldsymbol{Q}=(\pi, \pi)$. Here, we investigate whether a similar superconducting state may be stable also on a triangular lattice. However, we do not assume any particular value of $\boldsymbol{Q}$. Instead, we determine this quantity from the minimum of an appropriate thermodynamic potential. In the momentum representation one gets

$$
\begin{aligned}
H_{\mathrm{MF}} & =\sum_{\boldsymbol{k} \sigma}\left(E_{\boldsymbol{k}}-\mu\right) c_{\boldsymbol{k} \sigma}^{\dagger} c_{\boldsymbol{k} \sigma}-\frac{J E_{Q}}{t} \sum_{\boldsymbol{k}}\left(\Delta_{0}^{*} c_{-\boldsymbol{k}+\boldsymbol{Q} \downarrow} c_{\boldsymbol{k} \uparrow}+\text { h.c. }\right) \\
& +\frac{J\left|\Delta_{0}\right|^{2} E_{\boldsymbol{Q}} N}{t},
\end{aligned}
$$

where $E_{\boldsymbol{k}}=-2 t\left[\cos \left(k_{x}\right)+2 \cos \left(k_{x} \frac{1}{2}\right) \cos \left(k_{y} \frac{\sqrt{3}}{2}\right)\right]$. Let us note that the effective pairing interaction $U_{\text {eff }}=-J E_{\boldsymbol{Q}} / t$ depends on the momentum of the paired electrons. The mean-field Hamiltonian can be diagonalized with the help of the Bogoliubov transformation. The resulting thermodynamic potential $\Omega=-k T \ln \operatorname{Tr} \exp (-H / k T)$ reads

$$
\Omega=-k T \sum_{\boldsymbol{k}, z \in\{+,-\}} \ln \left(1+\exp \left(-\beta \lambda_{\boldsymbol{k} z}\right)\right)+\sum_{\boldsymbol{k}} E_{-\boldsymbol{k}+\boldsymbol{Q}}-N\left(\mu+U_{\mathrm{eff}}\left|\Delta_{0}\right|^{2}\right),
$$

where $\lambda_{\boldsymbol{k} \pm}=\frac{E_{\boldsymbol{k}}-E_{-\boldsymbol{k}+\boldsymbol{Q}}}{2} \pm \sqrt{\left(\eta_{\boldsymbol{k}}-\mu\right)^{2}+U_{\mathrm{eff}}^{2}\left|\Delta_{0}\right|^{2}}$ and $\eta_{\boldsymbol{k}}=\frac{E_{\boldsymbol{k}}+E_{-\boldsymbol{k}+\boldsymbol{Q}}}{2}$. From Eq. (4) one can easily obtain the average number of electrons $\langle n\rangle=-\frac{1}{N} \frac{\partial \Omega}{\partial \mu}$ as well as the gap equation $\frac{\partial \Omega}{\partial \Delta_{0}}=0$.

\section{Numerical results and discussion}

We have solved the gap equation and calculated the resulting potential $\Omega$ for various $\boldsymbol{Q}$. The left part in Fig. 1 shows that the superconducting transition temperature $T_{\mathrm{c}}$ is the largest, when the total momentum of the paired electrons approaches the edges of the first Brillouin zone (FBZ). More precisely, $T_{\mathrm{c}}$ is maximal when $\boldsymbol{Q}$ is equal to one of the momenta, which represent the corners of the FBZ (points A shown in the right part of Fig. 2). This result resembles the properties of the $\eta$-pairing on a square lattice, when $\boldsymbol{Q}=(\pi, \pi)$. The corresponding superconducting phase is thermodynamically stable since $\Omega\left(\Delta_{0} \neq 0\right) \leq \Omega(0)$, which can be 

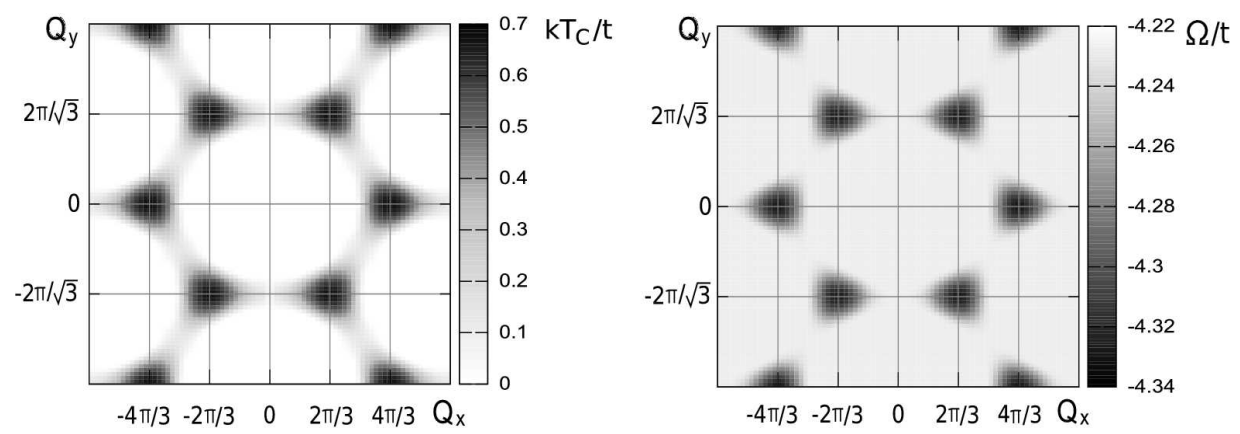

Fig. 1. Left part: $k T_{\mathrm{c}}$ as a function $Q$. Right part: $\Omega$ calculated for $k T=0.1 t$ and for $\Delta_{0}$ that has been determined from the gap equation. $J=1.87 t$ and $\mu=2.0 t$.
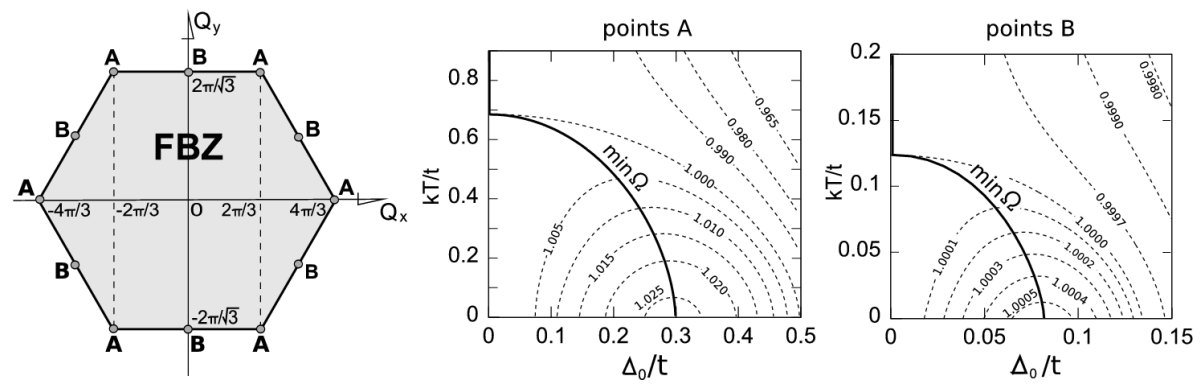

Fig. 2. $\Omega\left(\Delta_{0}\right) / \Omega(0)$ calculated for $J=1.87 t$ and $\mu=2.0 t$. The values of this ratio are indicated as labels to isolines. Two values of $\boldsymbol{Q}$ (denoted as A and B) are explicitly pointed out in the left part. The line labeled as $\min \Omega$ shows $\Delta_{0}$ that minimizes $\Omega$.

inferred from the right part in Fig. 1. One can see that $\Omega$ achieves the global minimum for the same values of $\boldsymbol{Q}$, when the transition temperature is maximal. We have found that the above results are qualitatively independent of the chemical potential. Although the magnitudes of the superconducting transition temperature and the superconducting order parameter depend of the concentration of electrons, the total momentum of the paired electrons remains doping independent. Therefore, we restrict the following analysis only to a few particular values of $\boldsymbol{Q}$. In Fig. 2 we show the isolines of the ratio $\Omega\left(\Delta_{0}, k T\right) / \Omega(0, k T)$. Let us note that $\Omega(0, k T)$ is negative. For a fixed temperature, $\Omega\left(\Delta_{0}, k T\right)$ has a single minimum, which clearly indicates that transition to the superconducting states is continuous. Again we have found that the character of the phase transition does not depend on the chemical potential. The line labeled as $\min \Omega$ shows $\Delta_{0}$ that minimizes the potential $\Omega$. Consequently, this line shows the temperature dependence of the superconducting order parameter. One can see that this dependence is very similar to that obtained from the standard BCS theory.

In order to visualize the difference between the discussed above superconducting state on a triangular lattice and the $\eta$-pairing on the square lattice, we 


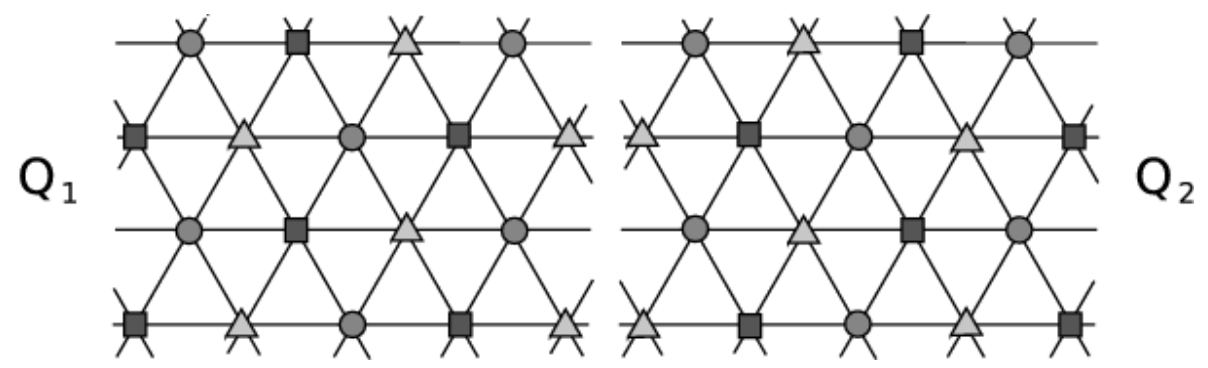

Fig. 3. Spatial dependence of $\Delta_{i}$ defined in Eq. (2) calculated for $\boldsymbol{Q}=\boldsymbol{Q}_{1}=\left(\frac{2}{3} \pi, \frac{2}{\sqrt{3}} \pi\right)$ and $\boldsymbol{Q}=\boldsymbol{Q}_{2}=\left(\frac{4}{3} \pi, 0\right)$, when $\Omega$ achieves the global minimum. Circles, squares, and triangles correspond to $\Delta_{i}=\Delta_{0}, \Delta_{i}=\Delta_{0} \exp \left(i \frac{2}{3} \pi\right)$ and $\Delta_{i}=\Delta_{0} \exp \left(-i \frac{2}{3} \pi\right)$, respectively.

have determined the spatial dependence of $\Delta_{i}$. For the thermodynamically most stable solution, $\Delta_{i}$ takes on three distinct values, as it is shown in Fig. 3. Although this spatial dependence visibly differs from the case of $\eta$-pairing, one can find some common properties: When the pair and single electron hopping interactions involve different sublattices, the phase of the superconducting order parameter is different for each of the sublattices. In the case of bipartite square lattice the system splits into two sublattices, whereas for triangular lattices there are three sublattices. Therefore, in the repulsive Penson-Kolb model the total momentum of electrons forming the Cooper pairs strongly depends on the geometry of the system.

\section{Acknowledgments}

This work was supported by the Polish Ministry of Education and Science under grant No. 1 P03B 07130.

\section{References}

[1] K.A. Penson, M. Kolb, Phys. Rev. B 33, 1663 (1986).

[2] S. Robaszkiewicz, B.R. Bułka, Phys. Rev. B 59, 6430 (1999).

[3] W.R. Czart, S. Robaszkiewicz, Phys. Rev. B 64, 104511 (2001).

[4] S. Robaszkiewicz, W.R. Czart, Phys. Status Solidi B 236, 416 (2003).

[5] M. Mierzejewski, M.M. Maśka, Phys. Rev. B 69, 054502 (2004).

[6] C.N. Yang, Phys. Rev. Lett. 63, 2144 (1989). 